Héctor Cuenya ${ }^{*}$ Departamento de Matemáticas, Universidad Nacional de Río Cuarto, 5.800 Río Cuarto, Argentina e-mail: hcuenya@@unrccc.edu.ar, mmarano@@unrccc.edu.ar

Miguel Marano, Departamento de Matemáticas, Universidad de Jaén, 23071 Jaén, Spain e-mail: mmarano@@jabalcuz.ujaen.es

\title{
A CHARACTERIZATION OF ORLICZ FUNCTIONS PRODUCING AN ADDITIVE PROPERTY
}

\begin{abstract}
It is shown that the only Luxemburg functionals that satisfy a very simply formulated property are induced by $p$ th-power functions, $0<p<$ $\infty$. The known result that Orlicz spaces cannot be normed analogously to $L_{p}$-spaces follows as a consequence.
\end{abstract}

\section{Introduction}

Let $\Psi:[0, \infty) \rightarrow[0, \infty]$ be a nondecreasing function, $\Psi(0)=0, \Psi(x) \rightarrow \infty$ as $x \rightarrow \infty$, and such that if $0<a<b, 0<\Psi(a), \Psi(b)<\infty$, then $\Psi$ is strictly increasing on $[a, b]$ and continuous on $[0, b]$. Such a function is called an $O$ function. Let $(\Omega, \mathcal{A}, \mu)$ be a measure space. Identify real valued functions on $\Omega$ that differ only on a set of measure zero. Let $\mathcal{M}$ denote the corresponding set of congruence classes. It is known $[2,3]$ that the pair $\{\Psi, \mu\}$ induces the Luxemburg functional on the Orlicz space

$$
\mathcal{L}_{\Psi}(\mu):=\left\{f \in \mathcal{M}: \int_{\Omega} \Psi(\alpha|f|) d \mu<\infty \text { for some } \alpha>0\right\} .
$$

Key Words: Orlicz function, Luxemburg norm, $L_{p}$-spaces

Mathematical Reviews subject classification: Primary: 46E30

Received by the editors August 27, 1995

* Partially supported by CONICOR and Univ. Nac. de Río Cuarto, Argentina. 
Its expression is

$$
\rho_{\Psi, \mu}(f):=\inf \left\{\lambda>0: \int_{\Omega} \Psi(|f| / \lambda) d \mu \leq 1\right\} .
$$

A well known example is the $L_{p}$-norm (with abuse of language for $0<p<1$ ), induced by any $\mu$ and $\Psi(x) \equiv c x^{p}, c>0$, for $0<p<\infty$, which gives $\mathcal{L}_{\Psi}(\mu)=L_{p}(\mu)=\left\{f \in \mathcal{M}: \int_{\Omega}|f|^{p} d \mu<\infty\right\}, \rho_{\Psi, \mu}(f)=\left(c \int_{\Omega}|f|^{p} d \mu\right)^{1 / p}$, and induced by any $\mu$ and a function $\Psi$ that satisfies $\Psi(x)=0$ if $0 \leq x \leq a$, $\Psi(x)=\infty$ if $x>a, a>0$, for $p=\infty$, which gives $\mathcal{L}_{\Psi}(\mu)=L_{\infty}(\mu)=$ $\{f \in \mathcal{M}$ : ess $\sup |f|<\infty\}, \rho_{\Psi, \mu}(f)=\|f\|_{\infty}=a$ ess $\sup |f|$. The $L_{p}$-norms satisfy the following property, applicable to any functional $\rho$ defined on a quite arbitrary real function space.

$$
\begin{aligned}
& \text { For any set } B \in \mathcal{A} \text { and } f, g \text { simple functions, if } \rho\left(f \chi_{B}\right)=\rho\left(g \chi_{B}\right) \\
& \text { and } \rho\left(f \chi_{\Omega \backslash B}\right)=\rho\left(g \chi_{\Omega \backslash B}\right) \text {, then } \rho(f)=\rho(g) .
\end{aligned}
$$

We recall that a simple function is one of form $\sum_{i=1}^{n} c_{i} \chi_{A_{i}}, \mu\left(A_{i}\right)<\infty$, where $\chi_{A}$ denotes the characteristic function of the set $A$. If $A$ has, exactly, none, one or two disjoint sets of finite and positive measure, then the class of all simple functions can be identified with $\{0\}, \mathbb{R}$ or $\mathbb{R}^{2}$, respectively, and in these cases any homogeneous functional defined on $\mathcal{L}_{\Psi}(\mu)$, depending on $|f|$, (e.g. a Luxemburg functional) satisfies $(*)$. We show in this paper that a rather different result follows when $\mathcal{A}$ has at least three disjoint sets of finite and positive measure $\mu$. The function $\Psi$ is said to satisfy property $\mathrm{P}_{\mu}$ if $\{\Psi, \mu\}$ induces a Luxemburg functional on $\mathcal{L}_{\Psi}(\mu)$ satisfying $(*)$. We shall give a description of such functions. In all cases they yield a $L_{p}$-norm, $0<p \leq \infty$. In case that $\mu$ is $\sigma$-finite and $\Psi$ is convex, this latter result can also be obtained from a classical theorem of H. F. Bohnenblust [1, 4]. As a consequence of that theorem, for $\operatorname{dim} \mathcal{L}_{\Psi}(\mu) \geq 3$ it is obtained that homogeneous functionals on $\mathcal{L}_{\Psi}(\mu)$ that satisfy $(*)$ are $p$-additive, $0<p \leq \infty$. For $p \geq 1$ this fact implies in turn that $\rho_{\Psi, \mu}(f)$ is a $L_{p}$-norm. However we do not follow the ideas of that theorem neither use the $p$-additive condition. Depending on a general measure $\mu$, in each case our proofs directly lead to the characterization of $\Psi$.

We consider in Section 2 a Luxemburg functional induced by a continuous $O$-function $\Psi$. In Section 3 we assume that $\Psi$ is not continuous and that $\mu$ is in addition a $\sigma$-finite measure. As a consequence of Theorem 1 we get in Section 4 the known fact that the space $\mathcal{L}_{\Psi}^{\prime}(\mu):=\left\{f \in \mathcal{M}: \int_{\Omega} \Psi(\alpha|f|) d \mu<\infty\right.$ for all $\alpha>0\}$ cannot be normed analogously to $L_{p}$-spaces, $p>0$, whenever $\operatorname{dim} \mathcal{L}_{\Psi}^{\prime}(\mu) \geq 2$.

We say that $(\Omega, \mathcal{A}, \mu)$ is infinitely divisible if there are measurable subsets of $\Omega$ with positive and arbitrarily small measure. If $(\Omega, \mathcal{A}, \mu)$ is not infinitely 
divisible and $\mathcal{A}$ has at least one set of finite and positive measure, then we let

$$
r_{0}=\inf \{\mu(A), A \in \mathcal{A}, \mu(A)>0\}>0 .
$$

\section{Characterization of a Continuous $O$-function $\Psi$}

In this section we assume that $\Psi$ satisfies $\Psi\left(\mathbb{R}_{+}\right) \supseteq \mathbb{R}_{+}$. Hence its right inverse function $\Psi^{-1}:(0, \infty) \rightarrow \mathbb{R}_{+}$exists, is continuous and satisfies $\Psi\left(\Psi^{-1}(x)\right)=x$ for all $x>0$. We say that a Luxemburg functional induced by any measure and such a function $\Psi$ is a $L$-functional.

Theorem 1 Assume that $\mathcal{A}$ has at least three disjoint sets of finite and positive measure.

(a) If $(\Omega, \mathcal{A}, \mu)$ is infinitely divisible, then $\Psi$ satisfies property $\mathrm{P}_{\mu}$ if and only if $\Psi(x) \equiv c x^{p}$ on $[0, \infty), c>0, p>0$.

(b) If $(\Omega, \mathcal{A}, \mu)$ is not infinitely divisible, then $\Psi$ satisfies property $\mathrm{P}_{\mu}$ if and only if $\Psi$ verifies $\Psi(x)=c x^{p}$ for any $x \in\left[0, \Psi^{-1}\left(1 / r_{0}\right)\right], p>0, c>0$.

In both cases the functional induced is a $L_{p}$-norm and therefore these are the only L-functionals that verify property $(*)$.

Proof. Assume that $\Psi$ satisfies property $\mathrm{P}_{\mu}$. Take $F \in \mathcal{A}, 0<\mu(F)<\infty$, such that there are two disjoint sets $G$ and $E$ of finite and positive measure, $G \cup E \subseteq \Omega \backslash F, \mu(E) \geq \mu(F)$. Such a set $F$ always exists due to the assumptions on $(\Omega, \mathcal{A}, \mu)$. Assume first $\mu(F)=\Psi(1)=1$. Take $h \in \mathbb{R}, h>0$, such that $\rho_{\Psi, \mu}\left(h \chi_{F \cup G}\right)=\rho_{\Psi, \mu}\left(\chi_{F}\right)=1$. So property $(*)$ implies $\rho_{\Psi, \mu}\left(h \chi_{F \cup G}+s \chi_{E}\right)=$ $\rho_{\Psi, \mu}\left(\chi_{F}+s \chi_{E}\right)=: \delta_{s}$ for any $s \in[0, \infty)$. Therefore, by definition of $\rho_{\Psi, \mu}$ and the continuity of $\Psi$ (on $[0,1)$ ), we get $\mu(F \cup G) \Psi(h)=1$ on the one hand and on the other hand

$$
\mu(F \cup G) \Psi\left(h / \delta_{s}\right)+\mu(E) \Psi\left(s / \delta_{s}\right)=\Psi\left(1 / \delta_{s}\right)+\mu(E) \Psi\left(s / \delta_{s}\right)=1,
$$

whence $\Psi\left(h / \delta_{s}\right)=\Psi(h) \Psi\left(1 / \delta_{s}\right)$ for any $s \geq 0$. As $s \mapsto \delta_{s}$ maps continuously $[0, \infty)$ onto $[1, \infty)$, we have obtained that $h$ is a multiplier for $\Psi$, i.e., $h$ is a point $m \in[0,1]$ that satisfies

$$
\Psi(m \gamma)=\Psi(m) \Psi(\gamma) \text { for any } \gamma \in[0,1] .
$$

Observe that the former equation implies that $\Psi\left(h^{n}\right)=[\Psi(h)]^{n}$ for $n \in \mathbb{N}$. Moreover, $h^{n}$ is a multiplier for $\Psi$. Since $0<h<1,0<\Psi(h)<1$, it follows that $h^{n} \downarrow 0, \Psi\left(h^{n}\right) \downarrow 0$ as $n \rightarrow \infty$ (and therefore $\Psi(x)>0$ if $x>0$ ). Take 
$k_{1} \in \mathbb{R}, k_{1}>0$, such that $\rho_{\Psi, \mu}\left(k_{1} \chi_{F}+m k_{1} \chi_{G}\right)=\rho_{\Psi, \mu}\left(\chi_{F}\right)=1$, where $m$ is a multiplier for $\Psi$. Hence $\Psi\left(k_{1}\right)+\mu(G) \Psi\left(m k_{1}\right)=1$. As $k_{1}<1$, it follows that

$$
\Psi\left(k_{1}\right)[1+\mu(G) \Psi(m)]=1 .
$$

On the other hand, property $(*)$ implies $\rho_{\Psi, \mu}\left(k_{1} \chi_{F}+m k_{1} \chi_{G}+s \chi_{E}\right)=\delta_{s}$ for any $s \geq 0$, whence $\Psi\left(k_{1} \gamma\right)=\Psi\left(k_{1}\right) \Psi(\gamma)$ for $\gamma \in[0,1]$. We have just proved that if $m$ is a multiplier for $\Psi$, then $k_{1}=\Psi^{-1}(1 /[1+\mu(G) \Psi(m)])$ is also a multiplier for $\Psi$. It follows that

$$
m_{n}=m_{n}(\Psi, \mu):=\Psi^{-1}\left(1 /\left[1+\mu(G) \Psi\left(h^{n}\right)\right]\right)
$$

is a multipler for $\Psi, n \in \mathbb{N}$. As $\Psi^{-1}$ is continuous and $\Psi^{-1}(1)=1$, we get that $m_{n} \uparrow 1$ as $n \rightarrow \infty$.

The existence of the sequence $\left\{m_{n}\right\}$ implies that any $m \in[0,1]$ is in the collection $\mathcal{P}$ of multipliers for $\Psi$. Indeed, observe first that $\mathcal{P}$ is closed because $\Psi$ is continuous. Hence

$$
\beta_{0}(m):=\inf \{\beta \in \mathcal{P}: \beta>m\} \in \mathcal{P} \text { for } m \in[0,1),
$$

and $\beta_{0}(m)=m$ because $m_{n} \beta_{0}(m) \in \mathcal{P}$ for all $n \in \mathbb{N}$.

For $x \in(0,1]$ we have

$$
\left[\Psi\left(x m_{n}\right)-\Psi(x)\right] /\left[x\left(m_{n}-1\right)\right]=\Psi(x)\left[\Psi\left(m_{n}\right)-\Psi(1)\right] /\left[x\left(m_{n}-1\right)\right] .
$$

On the other hand, the obvious estimates below show that $\Psi$ is absolutely continuous on $[\eta, 1]$ for all $\eta \in(0,1)$.

Let $x_{i} \in[\eta, 1], 1 \leq i \leq n+1, n \in \mathbb{N}$, and $x_{1}<x_{2}<\cdots<x_{n+1}$. Let $\gamma_{i}:=x_{i} / x_{i+1}, 1 \leq i \leq n$. Then

$$
\begin{aligned}
\eta \sum_{i=1}^{n}\left[1-\gamma_{i}\right] & <\sum_{i=1}^{n} x_{i+1}\left[1-\gamma_{i}\right]=\sum_{i=1}^{n}\left[x_{i+1}-x_{i}\right], \\
\sum_{i=1}^{n}\left[\Psi\left(x_{i+1}\right)-\Psi\left(x_{i}\right)\right] & =\sum_{i=1}^{n}\left[\Psi\left(x_{i+1}\right)-\Psi\left(\gamma_{i} x_{i+1}\right)\right] \\
& =\sum_{i=1}^{n} \Psi\left(x_{i+1}\right)\left[1-\Psi\left(\gamma_{i}\right)\right] \leq \sum_{i=1}^{n}\left[1-\Psi\left(\gamma_{i}\right)\right], \\
1-\Psi(\gamma) & \leq K[1-\gamma] \text { for some } K>0 \text { and any } \gamma<1 .
\end{aligned}
$$

Therefore we get that the derivative $\Psi^{\prime}(x)$ exists and is finite-valued for almost every $x$ on $[0,1]$. Hence the left side in eq. (1) converges to $\Psi^{\prime}(x)$ as $n \rightarrow \infty$ for 
almost every $x \in[0,1]$, and it follows that the right side in eq. (1) converges to $p \Psi(x) / x$ as $n \rightarrow \infty$ for any $x \in(0,1]$, where $p \geq 0$. Therefore $\Psi^{\prime}(x) / \Psi(x)=$ $p / x$ a.e. on $(0,1]$. As $\Psi$ is not constant, we have $p>0$. Since $\ln \Psi$ is absolutely continuous on $[\eta, 1]$, the integration of both sides of the former equation from $x$ to 1 gives $\Psi(x) \equiv x^{p}$ on $[0,1]$. Observe that, so far, only the restriction of $\Psi$ on $[0,1]$ has been considered (cf. the end of Section 3 ).

Suppose now $\mu(F)=r>0, \Psi(1) \geq 0$. The $L$-functional induced by $r \Psi$ and $\mu / r$ on $\mathcal{L}_{\Psi}(\mu)$ coincides with the $L$-functional induced by $\Psi$ and $\mu$. On the other hand, the $L$-functional induced by $\tilde{\Psi}(x):=r \Psi\left(\Psi^{-1}(1 / r) x\right)$ and $\mu / r$ on $\mathcal{L}_{\Psi}(\mu)$ is $\Psi^{-1}(1 / r)$ times the $L$-functional induced by $r \Psi$ and $\mu / r$, and therefore it also satisfies property $(*)$, with $\tilde{\Psi}(1)=(\mu / r)(F)=1$. So we get $\tilde{\Psi}(x) \equiv x^{p}$ on $[0,1]$, with $p>0$, whence $\Psi(x) \equiv c x^{p}$ on $\left[0, \Psi^{-1}(1 / r)\right]$, where $c=1 /\left[r\left(\Psi^{-1}(1 / r)\right)^{p}\right]$.

Under the hypothesis of (a) we can take $r \downarrow 0$. Then the case $\Psi^{-1}(1 / r) \uparrow b$, $b<\infty$, leads to a contradiction, whence $\psi^{-1}(1 / r) \uparrow \infty$ and the necessary part of (a) follows. The sufficiency of (a) is obvious. The necessity of (b) follows by taking $r \rightarrow r_{0}$. (Observe that this taking of limits in $r$ is compatible with the assumption on $F$ at the beginning of the proof). Conversely, if $\Psi(x) \equiv c x^{p}$ on $\left[0, \Psi^{-1}\left(1 / r_{0}\right)\right]$, then $\tilde{\Psi}(x):=r_{0} \Psi\left(\Psi^{-1}\left(1 / r_{0}\right) x\right) \equiv x^{p}$ on $[0,1]$ and, as mentioned above, $\tilde{\Psi}$ and $\mu / r_{0}$ induce, up to a multiplicative constant, the same $L$-functional as $\Psi$ and $\mu$. So, to conclude the proof, it suffices to observe that if $\Psi(x) \equiv x^{p}$ on $[0,1]$ and in addition $\mu(C) \geq 1$ for any measurable set $C$ with $\mu(C)>0$, then $\{\Psi, \mu\}$ induces the standard $L_{p}$-norm on $\mathcal{L}_{\Psi}(\mu)$ $\left(=L_{p}(\mu)\right)$. Indeed, if $g \in \mathcal{M}$ and $\int_{\Omega}|g| d \mu \leq 1$, then $|g| \leq 1$ almost everywhere $(\mu)$ on $\Omega$, whence $\int_{\Omega} \Psi(|f| / \lambda) d \mu \leq 1$ is equivalent to $\int_{\Omega}(|f| / \lambda)^{p} d \mu \leq 1$. Therefore $\rho_{\Psi, \mu}(f)=\inf \left\{\lambda: \int_{\Omega}(|f| / \lambda)^{p} d \mu=1\right\}=\left(\int_{\Omega}|f|^{p} d \mu\right)^{1 / p}$.

\section{Characterization of a Discontinuous $O$-function $\Psi$}

Now we suppose, and only in this section, that $\Psi$ is not $(\Omega, \mathcal{A}, \mu)$ is in addition a $\sigma$-finite measure space. So we consider an $O$-function $\Psi$ jumping to infinity at $a, a>0$. We say that the Luxemburg functional induced by such a pair $\{\Psi, \mu\}$ on $\mathcal{L}_{\Psi}(\mu)$ is a $L^{*}$-functional. Observe that when $(\Omega, \mathcal{A}, \mu)$ is not infinitely divisible, a $L^{*}$-functional may coincide with a $L$-functional. Since $\mu$ is $\sigma$-finite, the condition required to $\mathcal{A}$ in Theorem 1 is now equivalent to $\operatorname{dim} \mathcal{L}_{\Psi}(\mu) \geq$ 3. We can suppose without loss of generality that $\Psi$ is left continuous. An example of such a function is: $\Psi(x)=0$ if $0 \leq x \leq a, \Psi(x)=\infty$ if $x>a$. It is easy to see that $\{\Psi, \mu\}$, with this function $\Psi$, induces the a essential sup norm on $\mathcal{L}_{\Psi}(\mu)$, which we call, as usual, a $L_{\infty}$-norm. Moreover, it is easy to show that if $\mu(\Omega) \Psi(a) \leq 1$, then $\{\Psi, \mu\}$ induces a $L_{\infty}$-norm. Observe also 
that if $\mu(C) \geq 1$ for any measurable set $C$ of positive measure and $\Psi$ satisfies $\Psi(x) \equiv x^{p}$ on $[0,1]$ (e.g., $\Psi(x) \equiv x^{p}$ on $[0,1], \Psi(x)=\infty$ for $x>1$ ), then $\{\Psi, \mu\}$ induces the standard $L_{p}$-norm on $\mathcal{L}_{\Psi}(\mu)$.

Assume now that $\{\Psi, \mu\}$ induces a $L^{*}$-functional, where $\mu(\Omega) \Psi(a)>1$. Under this condition we consider two exhaustive cases. Suppose first that there exists $F \in \mathcal{A}, 0<\mu(F) \Psi(a)<1$. For instance, this is the case if $(\Omega, \mathcal{A}, \mu)$ is infinitely divisible. Assume also that there exists $B \in \mathcal{A}, B \supseteq F$, $\mu(B)<\mu(\Omega), \mu(B) \Psi(a)>1$. (This is the case if $\mu(\Omega)=\infty$ ). Then, since $\Psi$ is a continuous function on $[0, a)$, it follows that there exists $b, 0<b<a$, such that $\mu(F) \Psi(a)+\mu(B \backslash F) \Psi(b)=1$. Then we have $\rho_{\Psi, \mu}\left(a \chi_{F}\right)=\rho_{\Psi, \mu}\left(a \chi_{F}+\right.$ $\left.b \chi_{B \backslash F}\right)=1$. Take $E \in \mathcal{A}, E \subseteq \Omega \backslash B, 0<\mu(E)<\infty$. Therefore there exists $c, 0<c \leq a, \Psi(c)>0$, such that $\mu(F) \Psi(a)+\mu(E) \Psi(c) \leq 1$, whence $\rho_{\Psi, \mu}\left(a \chi_{F}+c \chi_{E}\right)=1$ but $\rho_{\Psi, \mu}\left(a \chi_{F}+b \chi_{B \backslash F}+c \chi_{E}\right)>1$. So property $(*)$ does not hold.

If for any set $D$ in $\mathcal{D}=\{D \in \mathcal{A}, D \supseteq F, \mu(D)<\mu(\Omega)\}$ is $\mu(D) \Psi(a) \leq 1$, then consider a set $B \in \mathcal{D}$ such that $\mu(B)=\sup \{\mu(D), D \in \mathcal{D}\}$. Hence

$\mu(B)<\mu(\Omega)<\infty$ and $\Omega \backslash B$ is an atom, whence $B$ is not an atom. At this point we can assume that $F$ satisfies at least one of the following conditions.

(1) There exists $G \in \mathcal{A}$ such that $F \subseteq G, \mu(F) \Psi(a)<\mu(G) \Psi(a)<1$.

(2) $F$ is an atom.

In either case it follows that $\mu(F)<\mu(B)$. We have $\rho_{\Psi, \mu}\left(a \chi_{F}\right)=\rho_{\Psi, \mu}\left(a \chi_{B}\right)=$ 1. As $\mu(F)+\mu(\Omega \backslash B)<\mu(\Omega)$, we get that $F \cup(\Omega \backslash B) \in \mathcal{D}$, i.e., $\mu(F) \Psi(a)+$ $\mu(\Omega \backslash B) \Psi(a) \leq 1$. So $\rho_{\Psi, \mu}\left(a \chi_{F}+a \chi_{\Omega \backslash B}\right)=1$ but $\rho_{\Psi, \mu}\left(a \chi_{B}+a \chi_{\Omega \backslash B}\right)>1$, whence property $(*)$ does not hold.

It remains to consider the case where $(\Omega, \mathcal{A}, \mu)$ is not infinitely divisible and $r_{0} \Psi(a) \geq 1$. In this case $\Psi^{-1}$ is well defined and continuous on the interval $\left(0,1 / r_{0}\right]$, whence the proof in Theorem $1(\mathrm{~b})$ applies without changes. Thus, in this case $\Psi$ satisfies property $\mathrm{P}_{\mu}$ if and only if $\Psi(x) \equiv c x^{p}$ on $\left[0, \Psi^{-1}\left(1 / r_{0}\right)\right]$. So we have the following.

Theorem 2 If $\operatorname{dim} \mathcal{L}_{\Psi}(\mu) \geq 3$, then the $L^{*}$-functional induced by $\Psi$ and $\mu$ satisfies property $(*)$ if it is necessarily a $L_{p}$-norm, $0<p \leq \infty$.

\section{$4 \mathcal{L}_{\Psi}^{\prime}(\mu)$ cannot be normed analogously to $L_{p}$-spaces}

Let $\mathcal{L}_{\Phi}^{\prime}(\mu)=\left\{f \in \mathcal{M}: \int_{\Omega} \Phi(a|f|) d \mu<\infty\right.$ for all $\left.\alpha>0\right\}$, where $\Phi$ is a finitevalued convex $O$-function. $\mathcal{L}_{\Phi}^{\prime}(\mu)$ is a linear subspace of $\mathcal{L}_{\Phi}(\mu)$. A natural way for trying to provide $\mathcal{L}_{\Phi}^{\prime}(\mu)$ with a norm, analogously to the $L_{p}$-norm, is to consider the expression $\Phi^{-1}\left(\int_{\Omega} \Phi(|f|) d \mu\right)$. We refer to [6] for a historical survey of this and related questions. In this article it is proved that this 
attempt is possible only if $\Phi(x) \equiv c x^{p}$, in the case where $\mu$ is the Lebesgue measure on the real line. This result was later extended [5] to the linear space $\mathcal{L}_{\Psi}^{\prime}(\mu)$, where $\Psi$ is a finite-valued strictly increasing $O$-function and $(\Omega, \mathcal{A}, \mu)$ is such that $\operatorname{dim} \mathcal{L}_{\Psi}^{\prime}(\mu) \geq 2$. More precisely, it is proved in that paper that if $\varphi_{\Gamma, \Psi, \mu}(f):=\Gamma\left(\int_{\Omega} \Psi(|f|) d \mu\right)$ is a homogeneous functional on $\mathcal{L}_{\Psi}^{\prime}(\mu)$, being $\Gamma$ and $\Psi$ finite-valued strictly increasing $O$-functions, then $\Psi(x) \equiv \Psi(1) x^{p}$, $\Gamma(x) \equiv \Gamma(1) x^{1 / p}, p>0$. Next we prove that this result is a consequence of Theorem 1. Assume first that $\operatorname{dim} \mathcal{L}_{\Psi}^{\prime}(\mu)=2$. Therefore $\mathcal{L}_{\Psi}^{\prime}(\mu)$ can be identified with $\mathbb{R}^{2}=\left\{\left(x_{1}, x_{2}\right), x_{1}, x_{2} \in \mathbb{R}\right\}$, and where

$$
\varphi_{\Gamma, \Psi, \mu}\left(x_{1}, x_{2}\right)=\Gamma\left(a_{1} \Psi\left(\left|x_{1}\right|\right)+a_{2} \Psi\left(\left|x_{2}\right|\right)\right), a_{1}, a_{2}>0 .
$$

Assume that $\varphi_{\Gamma, \Psi, \mu}\left(x_{1}, x_{2}\right)$ is a homogeneous functional. For any $x>0$ we have

$$
\varphi_{\Gamma, \Psi, \mu}(x, 0)=\Gamma\left(a_{1} \Psi(x)\right)=x \varphi_{\Gamma, \Psi, \mu}(1,0)=x \Gamma\left(a_{1} \Psi(1)\right) .
$$

As $\varphi_{a \Gamma, \Psi, \mu}\left(=a \varphi_{\Gamma, \Psi, \mu}\right)$ is also a homogeneous functional for all $a>0$, we can suppose without loss of generality that $\Gamma\left(a_{1} \Psi(1)\right)=1$. Under this assumption, $a_{1} \Psi \equiv \Gamma^{-1}$. Define on $\mathbb{R}^{3}$ the homogeneous functional

$$
\begin{aligned}
\varphi^{\prime}\left(x_{1}, x_{2}, x_{3}\right) & =\varphi_{\Gamma, \Psi, \mu}\left(\varphi_{\Gamma, \Psi, \mu}\left(x_{1}, x_{2}\right), x_{3}\right) \\
& =\Gamma\left(a_{1} \Psi\left(\Gamma\left(a_{1} \Psi\left(\left|x_{1}\right|\right)+a_{2} \Psi\left(\left|x_{2}\right|\right)\right)\right)+a_{2} \Psi\left(\left|x_{3}\right|\right)\right) \\
& =\Gamma\left(a_{1} \Psi\left(\left|x_{1}\right|\right)+a_{2} \Psi\left(\left|x_{2}\right|\right)+a_{2} \Psi\left(\left|x_{3}\right|\right)\right)
\end{aligned}
$$

This functional is of the form $\varphi_{\Gamma, \Psi, \mu^{\prime}}$, where $\operatorname{dim} \mathcal{L}_{\Psi}^{\prime}\left(\mu^{\prime}\right)=3$. This fact shows that it suffices to consider a homogeneous functional $\varphi_{\Gamma, \Psi, \mu}$ defined on $\mathcal{L}_{\Psi}^{\prime}(\mu)$, $\operatorname{dim} \mathcal{L}_{\Psi}^{\prime}(\mu) \geq 3$. After dividing $\Gamma$ by $\Gamma(1)$ we can suppose $\Gamma(1)=1$. For any $f \in \mathcal{L}_{\Psi}^{\prime}(\mu)$ we have that $\varphi_{\Gamma, \Psi, \mu}(f)=1$ if and only if $\rho_{\Psi, \mu}(f)=\inf \{\lambda$ : $\left.\int_{\Omega} \Psi(|f| / \lambda) d \mu \leq 1\right\}=1$, and since these two functionals are homogeneous, it follows that $\varphi_{\Gamma, \Psi, \mu}(f)=\rho_{\Psi, \mu}(f)$ for all $f \in \mathcal{L}_{\Psi}^{\prime}(\mu)$. Note that $\operatorname{dim} \mathcal{L}_{\Psi}(\mu) \geq 3$ implies that there exist three measurable sets of finite and positive measure, since $\Psi$ is strictly increasing. As the simple functions belong to $\mathcal{L}_{\Psi}^{\prime}(\mu)$ and $\varphi_{\Gamma, \Psi, \mu}$ satisfies property $(*)$, we get that Theorem 1 implies that $\varphi_{\Gamma, \Psi, \mu}(f)=$ $\left(c \int_{\Omega}|f|^{p} d \mu\right)^{1 / p}$ for all $f \in \mathcal{L}_{\Psi}^{\prime}(\mu)$, and also $\Psi(x) \equiv c x^{p}$ in the case where $(\Omega, \mathcal{A}, \mu)$ is infinitely divisible, and $\Psi(x) \equiv c x^{p}$ on $\left[0, \Psi^{-1}\left(1 / r_{0}\right)\right]$ in the case where $(\Omega, \mathcal{A}, \mu)$ is not infinitely divisible, $c>0, p>0$. For $\epsilon>0$ define $\Gamma^{*}(x)=\Gamma(x / \epsilon) / \Gamma(1 / \epsilon)$. Then applying in the latter case the same conclusion to the homogeneous functional $\varphi_{\Gamma^{*}, \psi, \epsilon \mu}=[1 / \Gamma(1 / \epsilon)] \varphi_{\Gamma, \Psi, \mu}$, and taking $\epsilon \downarrow 0$, it follows that also in this case $\Psi(x)=c x^{p}$ for any $x$ in $[0, \infty)$. Therefore $\Gamma\left(c \int_{\Omega}|f|^{p} d \mu\right)=\left(c \int_{\Omega}|f|^{p} d \mu\right)^{1 / p}$ for all $f \in \mathcal{L}_{\Psi}^{\prime}(\mu)$, whence it is easy to show that $\Gamma(x) \equiv x^{1 / p}$. 


\section{References}

[1] H. F. Bohnenblust, An axiomatic characterization of $L_{p}$-spaces, Duke Math. J., 6 (1940), 627-640.

[2] M. A. Krasnoselskii and Ya. B. Rutikii, Convex functions and Orlicz spaces, P. Noordhoff, Groningen, 1961.

[3] W. Luxemburg, Banach function spaces, Thesis, Technische Hogeschool te Delft, 1955.

[4] P. Meyer-Nieberg, Banach Lattices, Springer Verlag, Berlin and New York, 1991.

[5] W. Wnuk, Orlicz spaces cannot be normed analogously to $L_{p}$-spaces, Indag. Math., 87(3) (1984) 357-359.

[6] A. C. Zaanen, Some remarks about the definition of an Orlicz space, Lecture Notes in Mathematics, 945, Springer Verlag, Berlin and New York, 1982, pp. 263-268. 